\title{
De Geração em Geração: A Violência Conjugal e as Experiências na Família de Origem
}

\author{
Patrícia Manozzo Colossi \\ Aline Riboli Marasca \\ Denise Falcke \\ Universidade do Vale do Rio dos Sinos \\ São Leopoldo, RS, Brasil
}

\begin{abstract}
RESUMO
Considerando os aspectos transgeracionais familiares e a relevância do tema da violência no relacionamento conjugal para diversas áreas do conhecimento, o presente estudo teve o objetivo de identificar possíveis associações entre violência conjugal e experiências da família de origem e verificar seu poder preditivo na perpetração de violência conjugal. Uma amostra de 300 sujeitos respondeu a um questionário de dados sociodemográficos, o Family Background Questionnaire e a Revised Conflict Tactics Scales. Os índices de violência conjugal mostraram-se expressivos e estabeleceram correlações significativas com as experiências de agressão na família de origem. Entre as mulheres, negligência física, abuso sexual e abuso de substâncias paterno foram preditores da agressão, enquanto, para os homens, o preditor foi abuso físico paterno. Discute-se o impacto das vivências na família de origem, assim como a relevância de políticas de atendimento a situações de violência, com vistas de interromper a perpetuação de um padrão relacional violento.
\end{abstract}

Palavras-chave: Violência conjugal; família de origem; transgeracionalidade; violência familiar.

\section{ABSTRACT}

\section{Generation by Generation: Marital Violence and Experiences in the Family of Origin}

Considering the family's transgenerational aspects and the relevant of theme for different knowledge fields, this study aims identifying possible associations between experiences in family-of-origin and intimate partner violence, as well as verifying the predictive power of early experiences in the perpetration of marital violence. A sample of 300 people answered a sociodemographic questionnaire, Family Background Questionnaire and Revised Conflict Tactics Scale. The data showed important correlations between abusive experience in the family-of-origin and intimate partner violence. Among women, physical neglect, sexual abuse and parental substance abuse were predictors of aggression committed in their relationships. While in male sample, the predictor was paternal physical abuse. This article discusses the impact of experiences in the family-of-origin, as well as the relevance of social services policies targeting couples and families in situations of violence and whose aim is to stop the perpetuation of violent relational patterns.

Keywords: Intimate partner violence; family-of-origin; intergenerational transmission; family violence.

\section{RESUMEN}

\section{De Generación en Generación: La Violencia Conjugal y las Experiencias en la Familia de Origen}

En vista de los aspectos transgeneracionales familiares y la pertinencia de la temática de la violencia de pareja para las distintas áreas de conocimiento, este estudio tiene como objetivo identificar possibles asociaciones entre la violencia conjugal y las experiencias en la família de origen y verificar su predicción en la ocurrencia de la violencia conjugal. Una mostra de 300 sujetos responderon a un cuestionario de datos demográficos, al Family Background Questionnaire y a la Revised Conflict Tactics Scales . Las tasas de violencia conjugal fueran expresivas y estabeleceron asociaciones significativas com las experiencias de agresión en la família de origen. Para las mujeres, la negligencia física, el abuso sexual y el abuso de sustancias por el padre fueran predictores de la agresión, mientras que, para los hombres, el predictor fue el abuso físico por el padre. Se discuten el impacto de las experiencias en la familia de origen, así como la pertinencia de políticas de tratamiento a las situaciones de violencia, en orden de interrumpir la perpetuación de un modelo de relación violento.

Palabras clave: Violencia conjugal; familia de origen; transgeneracionalidad; violencia familiar. 


\section{INTRODUÇÃO}

A violência conjugal tem sido tema de inúmeros estudos em todo o mundo, tanto por suas diversas formas de expressão, quanto pelas consequências para o casal e para a família como um todo. A Organização Mundial da Saúde ressalta a gravidade da questão e aponta a importância de uma articulação interdisciplinar a fim de prevenir e tratar os envolvidos neste fenômeno (Dahlberg \& Krug, 2007; Silva, Valadares, \& Souza, 2013). Serviços de saúde, jurídicos, policiais e de assistência social deparam-se com a crescente demanda de atendimento aos casos de violência entre parceiros íntimos, revelando a necessidade de formação e capacitação dos profissionais para intervirem nestas situações (Borsoi, Brandão, \& Cavalcanti, 2009; Cortez, Cruz, \& Souza, 2013, Gadoni-Costa, Zucatti, \& Dell'Aglio, 2011; Kiss \& Schraiber, 2011; Leal, Lopes, \& Gaspar, 2011).

Os dados de prevalência são diversos, conforme o contexto investigado. Resultados indicam que, ao redor do mundo, entre $22 \%$ e $49,5 \%$ das mulheres reportam algum episódio de abuso experienciado por parte de seus companheiros (Ansara \& Hindin, 2009; Fehringer \& Hindin, 2009; Jaoko, 2010; Stenson \& Heimer, 2008; Yoshihma \& Horrocks, 2010). No Brasil, os levantamentos estimam que entre $26 \%$ e $34,5 \%$ das mulheres sofrem violência física ou sexual por parte de seus companheiros (Bruschi, Paula, \& Bordin, 2006; Lamoglia \& Minayo, 2009; Miranda, Paula, \& Bordin, 2010; Vieira, Perdona, \& Santos, 2011).

Entre as publicações nacionais, identificam-se dados comumente provindos de serviços de saúde, delegacias da mulher, centros especializados em atendimento à vítima de violência ou de processos judiciais (Garcia et al., 2008; Lamoglia \& Minayo, 2009; Leôncio et al., 2008; Melo, Silva, \& Caldas, 2009; Vieira, Perdona, $\&$ Santos, 2011). Dessa forma, o acesso à informação costuma ser a partir de denúncia ou de ocorrência de um episódio de violência física, suprimindo os casos em que a violência não é revelada para além do contexto conjugal/familiar e se mantém como um segredo na família. Além disso, enfocam prioritariamente a violência sob uma perspectiva de gênero, uma vez que os serviços especializados no tratamento dos casos envolvendo violência, como delegacias da mulher e casas de acolhimento, centram a sua atuação no acolhimento da mulher vítima e no encaminhamento do agressor para punição ou tratamento de recuperação, prioritariamente relacionado ao abuso de substâncias químicas (Fernández-Montalvo, López-Goñi, \& Arteaga, 2011; Macy \& Goodbourn, 2012; Straus, 2010).
São escassas as propostas de intervenção com o casal envolvido no contexto violento, partindo de uma compreensão relacional, em que homem e mulher participam, em alguma medida, do estabelecimento e da manutenção da conjugalidade violenta. Um estudo brasileiro em fase de desenvolvimento, realizado no RS e conduzido por Falcke (2012), tem buscado prestar atendimento psicológico ao casal que vivencia a situação de violência considerando a possibilidade de reorganizar a estrutura conjugal e desenvolver novas estratégias de resolução de conflitos mais saudáveis e sem o uso da violência, em suas diferentes formas de expressão. Propostas de intervenção como esta buscam prevenir a perpetuação da violência conjugal como um modelo transgeracional, que atravessa gerações e se mantém como um padrão a ser seguido, naturalizado para muitas famílias.

Outros estudos seguem em direção semelhante e revelam a importância de favorecer a qualidade conjugal, como aspecto protetor do ambiente familiar, uma vez que, administrando os conflitos vivenciados pelo casal, observa-se uma reverberação positiva nos demais subsistemas familiares, favorecendo a saúde física e mental dos filhos (Mosmann, Zordan, \& Wagner 2011; Pedro, 2013; Villas Boas, Dessen, \& Melchiori, 2010). Nesta perspectiva, casais cujos relacionamentos são predominantemente disfuncionais apresentam maiores chances de perpetuarem um modelo relacional negativo, com diferentes expressões de violência.

A literatura enfatiza a complexidade do fenômeno da violência conjugal, apontando diversos fatores associados para a sua ocorrência, tais como: gênero (Dourado \& Noronha, 2014; Paixão, Gomes, Diniz, Couto, Vianna, \& Santos, 2014; Souto \& Braga, 2009); aspectos sociais (Lamoglia \& Minayo, 2009), características sociodemográficas (Zanoti-Jeronymo, Zaleski, Pinsky, Caetano, Figlie, \& Laranjeira, 2009), psicopatologias (Boyle, O'Leary, Rosenbaum, \& Hassett-Walker, 2008; Lawson \& Rivera, 2008), abuso de álcool e outras substâncias (Stickley, Timofeeva, \& Sparen, 2008; Whiting, Simmons, Havens, Smith, \& Oka, 2009), baixo suporte social (Banyard \& Modeki, 2006), ambiente comunitário violento (Koenig, Stephenson, Ahmed, Jejeebhoy, \& Campbell, 2006), exposição à violência conjugal na infância (Gover, Park, Tomsich \& Jennings, 2011) e experiências de vitimização na família de origem (Fang \& Corso, 2008). Destaca-se, ainda, os componentes sociais envolvidos no fenômeno, marcando uma cultura patriarcal com papéis rígidos que, certamente, contribuem para eclosão e manutenção do fenômeno da violência (Narvaz \& Koller, 2006 ). Dados de prevalência destacam o homem como principal vítima da violência em geral. Contudo, 
quando a vítima é mulher, estudos referem que $1 \mathrm{em}$ cada 3 mulheres e vítima de seu companheiro amoroso ao longo da vida (Lamoglia \& Minayo, 2009; Miranda, Paula, \& Bordin, 2010; Pinto, Moraes, \& Monteiro, 2015; Rosa, Boing, Büchele, Oliveira, \& Coelho, 2008; Vieira, Perdona, \& Santos, 2011).

Para a compreensão do fenômeno da violência conjugal, Carvalho-Barreto, Bucher-Maluschke, Almeida e DeSouza (2009) partem de uma compreensão que considera quatro níveis em interação, abrangendo características individuais, interações interpessoais, aspectos contextuais e perspectiva temporal, que inclui a transmissão transgeracional. Em relação a este último nível, destaca-se a importância da família de origem no comportamento aprendido e perpetuado de geração em geração. As crianças que experienciam contextos de violência na família de origem, como vítimas diretas ou como testemunhas da violência interparental, levam para seus relacionamentos futuros a tendência de repetição dos padrões vivenciados (Falcke, 2006; Mendlowicz \& Figueira, 2007; Paradis et al., 2009).

As consequências sofridas pela convivência em contextos familiares violentos podem ser diversas e podem apresentar-se de forma diferente para homens e mulheres, incluindo: psicopatologias, transtornos de comportamento, cometimento de atos infracionais e, de modo especial, envolvimento em relacionamentos íntimos violentos na vida adulta (Hughes, Stuart, Gordon, \& Moore, 2007; Lawson \& Rivera, 2008; Milner et al., 2010; Noll, Trickett, Harris, \& Putman, 2009; Silva, Menezes, \& Lopes, 2010; Durand, Schraiber, FrançaJunior, \& Barros, 2011; Weisbart et al., 2008). Estudos mostram que mulheres vítimas de abuso sexual, abuso físico, negligência e testemunhas da violência entre os pais, como expressão de violência na família de origem, mostram-se mais predispostas à vitimização em seus relacionamentos conjugais na vida adulta (Alexander, 2011; Fang \& Corso, 2008; Fergusson, 2011; Stickley, Timofeeva, \& Sparen, 2008; Yoshima \& Horrocks, 2010). Já os homens, quando vítimas de abuso físico e/ou sexual em suas famílias mostram-se mais predispostos a envolverem-se em relacionamentos amorosos como perpetradores da violência (Fang \& Corso, 2008; Fergusson, 2011; Padovani \& Williams, 2011).

Com isso, a experiência de violência vivenciada na família de origem impacta na vida do indivíduo não apenas em suas relações afetivas, como modelo de relacionamento amoroso, mas também em outros contextos, legitimando a violência como estratégia de resolução de conflitos nas mais diversas situações. Nesse sentido, aprofundar os estudos da referida temática, bem como ampliar as possiblidades de intervenção com o casal e com a família, podem expressar- se como potenciais para restaurar as relações familiares, e, quiçá, favorecer o rompimento do ciclo de violência conjugal através das gerações.

Partindo desse pressuposto, o objetivo do presente estudo foi identificar possíveis associações entre a violência conjugal e as experiências vivenciadas na família de origem. Além disso, buscou-se identificar o poder preditivo das experiências de violência na família de origem para a perpetração da violência conjugal.

\section{MÉTODO}

\section{Delineamento}

O presente estudo tem caráter quantitativo, com delineamento descritivo, correlacional e explicativo. A presente escolha metodológica pautou-se na necessidade de mapear o fenômeno da violência conjugal e sua associação com as experiências vividas na família de origem, considerando-as como possível fator preditor da eclosão do fenômeno da violência entre cônjuges.

\section{Participantes}

Participaram deste estudo 300 sujeitos (150 homens e 150 mulheres), casados ou em união estável, com tempo mínimo de união de seis meses, da região metropolitana de Porto Alegre, Rio Grande do Sul. A idade dos respondentes variou entre 19 e 81 anos, $(\mathrm{m}=41,17 ; \mathrm{dp}=12,75)$ e o tempo de união variou de 6 meses a 56 anos $(m=15,76 ; d p=12,06)$. Dos participantes, $205(68,33 \%)$ indicaram possuir filhos, sendo que o número médio de filhos foi de 1,8 . Os participantes foram inseridos no estudo por conveniência através da indicação de conhecidos. O critério de inclusão foi estar casado, oficialmente ou morando junto, por um tempo mínimo de seis meses.

\section{Instrumentos}

Para a coleta de dados foram aplicados os seguintes instrumentos:

- Questionário de dados sociodemográficos: composto por 19 questões que visam obter informações pessoais e dados acerca do relacionamento conjugal.

- Family Background Questionnaire (FBQ): desenvolvido por Melchert (1998a, 1998b) e traduzido para o português por Falcke (2003), foram utilizadas as subescalas de negligência física, abuso físico (paterno e materno), abuso sexual, ajustamento psicológico (paterno e materno), estilo de tomada de decisão (paterno e materno), aliança parental e abuso de substâncias (paterno e materno), a fim de investigar as experiências vividas na família de origem. Os alphas obtidos no estudo de Falcke (2003) foram de 0,99 para a escala total e os seguintes valores, conforme 
a subescala: Abuso de Substâncias pelo Pai $(0,9339)$, Estilo de Tomada de Decisão Paterno (0,8919), Aliança Parental $(0,8756)$, Estilo de Tomada de Decisão Materno $(0,8632)$, Ajustamento Psicológico Paterno $(0,8292)$, Abuso Físico Paterno $(0,8049)$, Negligência Física (0,8023), Ajustamento Psicológico Materno $(0,7022)$, Abuso de Substâncias pela Mãe $(0,6827)$, Abuso Físico Materno $(0,6492)$ e Abuso Sexual $(0,4069)$.

- Revised Conflict Tactics Scales (CTS2): concebida por Straus, Hamby, McCoy e Sugarman (1996) e adaptada à língua portuguesa por Moraes, Hasselmann e Reichenheim (2002), foi aplicada com a finalidade de identificar a presença de violência na vida conjugal. O referido instrumento é composto por cinco subescalas formadas por 78 itens que descrevem possíveis ações do respondente e do companheiro afetivo, abrangendo cinco dimensões: violência física, agressão psicológica, coerção sexual, lesão corporal e negociação. As dimensões de violência física, agressão psicológica, coerção sexual e lesão corporal são subdivididas em níveis de severidade grave ou menor. $\mathrm{O}$ alpha de Cronbach obtido para a CTS2 foi de 0,92 .

\section{Procedimentos de coleta dos dados}

A fim de atender às exigências éticas contempladas na resolução para pesquisas com seres humanos (Resolução 466/2012 do Conselho Nacional de Saúde e CFP 026/2000), esse estudo foi encaminhado para avaliação do Comitê de Ética da Universidade, tendo sua aprovação sob o parecer 11/129. Posteriormente, foram feitos contatos com os participantes convidando-os a participar do estudo.

Os dados foram coletados por uma equipe de pesquisa treinada para a aplicação dos instrumentos, realizada na residência dos participantes ou em local indicado por eles, na presença de um assistente de pesquisa. Inicialmente, foi fornecido um Termo de Consentimento Livre e Esclarecido (TCLE), indicando, de forma clara, aos casais, o objetivo do estudo, procedimentos, utilização dos dados obtidos e assegurando-os quanto à confidencialidade dos mesmos. Foi esclarecida a forma de utilização dos dados obtidos, bem como a ampla e irrestrita liberdade do aceite ou não na participação da pesquisa e a possibilidade de desistência em qualquer tempo. Os instrumentos foram respondidos simultaneamente, sem que os cônjuges tivessem acesso às informações fornecidas pelo outro. Ao término da aplicação, os questionários foram colocados em um envelope lacrado, entregues ao assistente de pesquisa para posterior análise.

\section{Análise dos dados}

Os dados foram analisados por meio do programa estatístico SPSS (versão 21.0), sendo realizadas análises descritivas, teste de Correlação de Pearson, para análise de associação entre as experiências na família de origem e a violência conjugal, e análise de regressão, método stepwise, para análise do poder preditivo das experiências na família de origem na ocorrência de violência conjugal.

\section{RESULTADOS}

Os resultados indicaram índices expressivos de violência conjugal na amostra pesquisada, a partir do CTS2, que variaram de $1,1 \%$ para coerção sexual grave a $86,6 \%$ para agressão psicológica menor, como se observa na Figura 1.

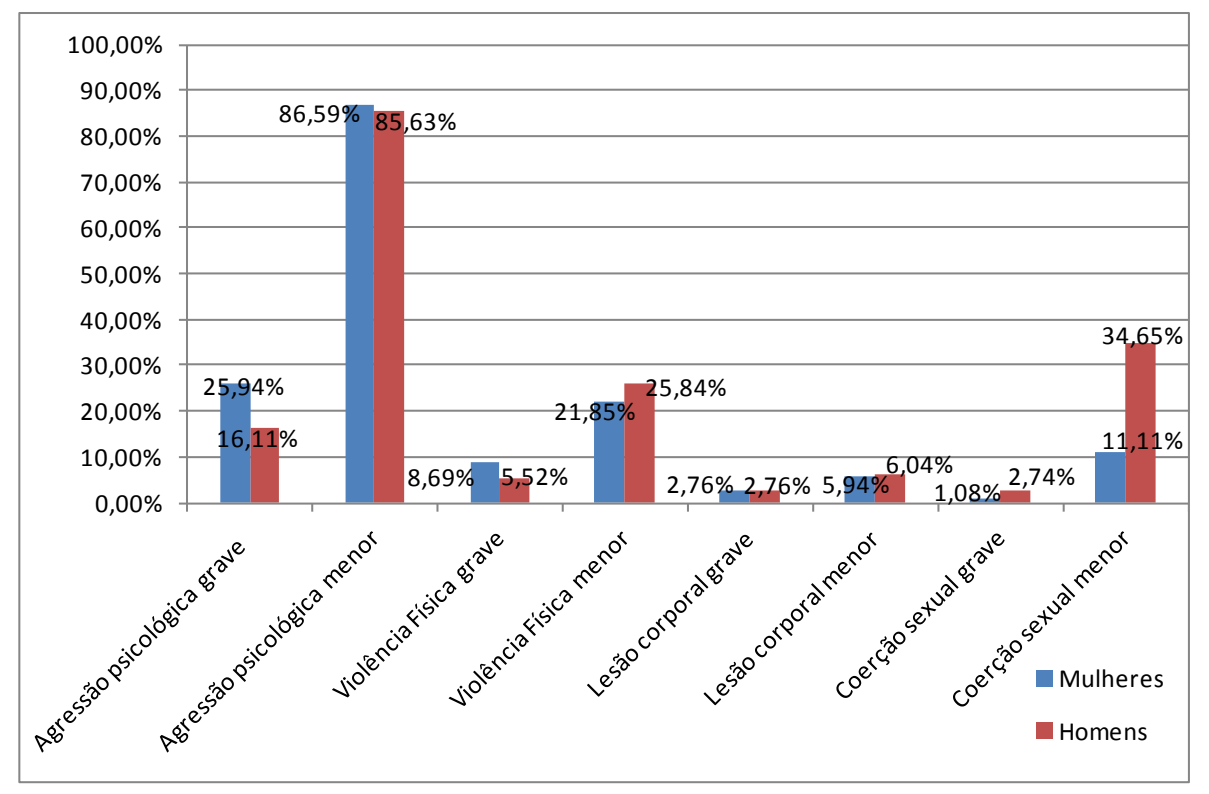

Figura 1. Porcentagem de ocorrência de violência conjugal conforme o sexo 
As categorias reveladas na Figura 1 são apresentadas pelo instrumento CTS2, que busca mensurar os tipos de violência conjugal a partir das dimensões: agressão psicológica maior e menor, violência física maior e menor, coerção sexual maior e menor, lesão corporal maior e menor e negociação. Observou-se diferença estatisticamente significativa, na comparação entre os sexos, em relação à coerção sexual menor $(\mathrm{p}<0,001$, $\left.\mathrm{x}^{2}=28,075\right)$, indicando que homens a cometem (34,65\%) mais do que as mulheres $(11,11 \%)$, e agressão psicológica grave $\left(\mathrm{p}=0,021, \mathrm{x}^{2}=5,301\right)$ apontando que mulheres a cometem $(25,94 \%)$ mais do que os homens $(16,11 \%)$. No que se refere às dimensões de lesão corporal e violência física não foram observadas diferenças estatísticas entre os sexos.

As associações entre as experiências vivenciadas na família de origem e a perpetração de violência no relacionamento conjugal atual são apresentadas na Tabela 1.

Observou-se um número elevado de correlações entre as experiências na família de origem e a violência conjugal, especialmente considerando as dimensões de abuso físico (materno e paterno), abuso sexual, ajustamento psicológico (paterno e materno) e aliança parental.

Buscando verificar o poder preditivo das variáveis da família de origem na ocorrência de violência conjugal, foi realizada uma análise de regressão. Considerando os dados da amostra feminina, a negligência física, o abuso sexual e o abuso de substâncias paterno mostraram-se preditores de violência cometida pelas mulheres e a aliança parental foi identificada como variável protetora (Tabela 2 ). Tal procedimento forneceu um coeficiente de variância explicada $\left(\mathrm{R}^{2}\right)$ de 0,323 , o que determina que essas variáveis explicam $32,3 \%$ da violência cometida pelas mulheres.

No caso da amostra masculina, somente o abuso físico paterno mostrou-se preditor da violência cometida no relacionamento conjugal pelos homens (Tabela 3), obtendo um coeficiente de variância explicada $\left(\mathrm{R}^{2}\right)$ de 0,111 , explicando $11,1 \%$ da violência cometida por eles.

TABELA 1

Correlações entre dimensões do FBQ e perpetração de violência do CTS2

\begin{tabular}{|c|c|c|c|c|c|c|}
\hline & $\begin{array}{c}\text { Coerção sexual } \\
\text { grave }\end{array}$ & $\begin{array}{c}\text { Coerção sexual } \\
\text { menor }\end{array}$ & $\begin{array}{c}\text { Violência fisica } \\
\text { grave }\end{array}$ & $\begin{array}{c}\text { Violência fisica } \\
\text { menor }\end{array}$ & $\begin{array}{c}\text { Agressão } \\
\text { psicológica grave }\end{array}$ & $\begin{array}{c}\text { Agressão } \\
\text { psicológica menor }\end{array}$ \\
\hline Abuso físico paterno & $0,147 *$ & 0,047 & $0,190 * *$ & $0,138 *$ & 0,087 & $0,176^{* *}$ \\
\hline Abuso físico materno & 0,098 & 0,030 & $0,165 * *$ & $0,151 *$ & $0,117 *$ & $0,184 * *$ \\
\hline Abuso sexual & 0,109 & $0,125^{*}$ & $0,174 * *$ & $0,211 * *$ & $0,191 * *$ & $0,268 * *$ \\
\hline Negligência física & $0,133 *$ & 0,057 & $0,132 *$ & $-0,018$ & 0,076 & 0,105 \\
\hline Estilo de decisão paterno & $-0,082$ & 0,038 & $-0,094$ & $-0,063$ & $-0,080$ & $-0,126^{*}$ \\
\hline Estilo de decisão materno & $-0,140^{*}$ & 0,051 & $-0,176^{* *}$ & $-0,090$ & $-0,115$ & $-0,095$ \\
\hline Abuso de substância paterno & 0,058 & 0,115 & 0,073 & 0,021 & 0,011 & 0,007 \\
\hline Abuso de substância materno & $-0,023$ & 0,035 & 0,047 & 0,123 & 0,100 & 0,113 \\
\hline Ajustamento psicológico paterno & $-0,159^{*}$ & $-0,048$ & $-0,197 * *$ & $-0,187 * *$ & $-0,195 * *$ & $-0,245^{* *}$ \\
\hline Ajustamento psicológico materno & 0,016 & 0,062 & $-0,181 * *$ & $-0,188 * *$ & $-0,190 * *$ & $-0,267 * *$ \\
\hline Aliança parental & 0,028 & $-0,008$ & $-0,148 *$ & $-0,134 *$ & $-0,194 * *$ & $-0,288 * *$ \\
\hline
\end{tabular}

TABELA 2

Experiências na família de origem preditoras de violência cometida contra o parceiro pelas mulheres

\begin{tabular}{lccccc}
\hline \multirow{2}{*}{\multicolumn{1}{c}{ Variáveis do modelo }} & \multicolumn{2}{c}{ Coeficientes não padronizados } & \multicolumn{3}{c}{ Coeficientes padronizados } \\
\cline { 2 - 6 } & $B$ & Modelo padrão & Beta & S &, 000 \\
\hline Aliança Parental &,- 142 &, 029 &,- 659 & $-4,902$ &, 000 \\
Negligência Física &, 183 &, 048 &, 469 & 3,828 &, 003 \\
Abuso Sexual & 1,598 &, 527 &, 311 & 3,034 &, 016 \\
Abuso de Substâncias Paterno &, 094 &, 038 &, 280 & 2,469 & \\
\hline
\end{tabular}

$\mathrm{R}=0,568 ; \mathrm{R}^{2}=0,323 ; \mathrm{R}^{2}$ ajustado $=0,286$.

TABELA 3

Experiências na família de origem preditoras de violência cometida contra a parceira pelos homens

\begin{tabular}{lccccc}
\hline \multirow{2}{*}{ Variáveis do modelo } & \multicolumn{3}{c}{ Coeficientes não padronizados } & \multicolumn{3}{c}{ Coeficientes padronizados } \\
\cline { 2 - 6 } & $B$ & Modelo padrão & Beta & S & Sig \\
\hline Abuso Físico Paterno &, 218 &, 072 &, 333 & 3,016 &, 004 \\
\hline
\end{tabular}

$\mathrm{R}=0,333 ; \mathrm{R}^{2}=0,111 ; \mathrm{R}^{2}$ ajustado $=0,099$. 
As experiências de negligência física $(\beta=0,469$; $\mathrm{p}<0,001)$, abuso sexual $(\beta=0,311 ; \mathrm{p}=0,003)$ e abuso de substâncias paterno $(\beta=0,280 ; p=0,016)$ das mulheres, bem como o abuso físico paterno sofrido pelos homens $(\beta=0,333 ; p=0,004)$, podem ser consideradas variáveis preditoras de violência cometida contra o parceiro íntimo. Os sujeitos que possuíam essas vivências obtiveram maiores índices de violência cometida na relação conjugal. Por outro lado, a experiência de melhor aliança parental percebida pelas mulheres $(\beta=-0,659$; $\mathrm{p}<0,001)$ foi considerada protetiva de comportamentos violentos contra o parceiro na relação conjugal.

\section{DISCUSSÃO}

De modo amplo, os resultados ratificaram estudos anteriormente realizados que revelavam associação entre experiências de violência na família de origem, como vítima ou como testemunha, e o envolvimento em uma conjugalidade violenta na vida adulta (Black, Sussman, \& Unger, 2010; Fehringer \& Hindin, 2009; Fergusson, 2011; Gover, Park, Tomsich, \& Jennings, 2011; Jaoko, 2010). De forma semelhante, a qualidade negativa de vínculo da dupla parental na infância parece relacionar-se, em alguma medida, com contextos violentos na conjugalidade adulta.

Os resultados obtidos no presente estudo destacam a associação entre as experiências de violência na família de origem como modelo relacional que pode se estabelecer em diferentes contextos da vida adulta, merecendo destaque os relacionamentos amorosos. Tomando a qualidade conjugal como fator de proteção do ambiente familiar, os conflitos vividos pelo casal, sem adequada administração, acabam reverberando nos outros subsistemas, podendo impactar na saúde física e mental dos filhos (Mosmann, Zordan \& Wagner, 2011, Wagner \& Goulart, 2013). Neste sentido, casais cuja relação está pautada em características disfuncionais revelaram maiores chances de apresentarem aos filhos um modelo de relação em que pesem diferentes expressões de violência. No sentido oposto, o favorecimento de modelos relacionais marcados pelo ajustamento conjugal, coesão e flexibilidade entre os pais oferece aos filhos um aprendizado transgeracional de uma conjugalidade mais adaptativa e funcional.

Em diversos estudos, o abuso sexual na infância foi identificado como fator preditor de perpetração e vitimização por violência no relacionamento conjugal na vida adulta das mulheres. O mesmo acontece com experiências de abuso físico, negligência e testemunho da violência entre os pais, predispondo as mulheres pesquisadas ao estabelecimento de relações conjugais violentas na vida adulta (Alexander, 2011; Fang \& Corso,
2008; Fergusson, 2011; Stickley, Timofeeva, \& Sparen, 2008; Yoshima \& Horrocks, 2010). Já para os homens, a literatura aponta o impacto do abuso físico e sexual vivenciado na perpetração da agressão nos relacionamentos íntimos (Fang \& Corso, 2008; Fergusson, 2011; Padovani \& Williams, 2011). No caso da amostra feminina, observou-se que um conjunto de variáveis da família de origem (negligência física, abuso sexual e abuso de substâncias paterno) mostrou-se preditor da agressão cometida por elas em seus relacionamentos. Além disso, verificou-se que o relacionamento interparental também impactou a experiência de violência perpetrada pelas mulheres, contra o parceiro, evidenciando o quanto elas mostram-se sensíveis ao que observam do relacionamento conjugal dos pais. Para os homens, a única experiência da família de origem identificada como preditora da violência conjugal foi o abuso físico paterno cometido diretamente contra eles, ratificando achados anteriores que apontaram o pai como principal agressor na infância e modelo que leva homens a cometerem violência contra sua parceira na vida adulta (Padovani \& Williams, 2011). Este resultado evidencia a possibilidade de identificação com o agressor, que os leva a perpetuação da violência.

Os resultados deste estudo lançam luz sobre qual o tipo de abuso que assume maior influência para que a agressão se perpetue nos casais adultos, sendo importante considerar o impacto que tais vivências geram nas crianças vitimizadas. As situações violentas dentro da família, por vezes, podem ser de difícil reconhecimento pelos próprios membros, tendo em vista que, apesar dos laços familiares incluírem a violência, ocorrem simultaneamente relações de carinho e dependência (Martins et al., 2007). Dessa forma, o modo de relação vivenciado e aprendido pelos indivíduos passa a conceber a agressão como algo naturalizado e inerente aos relacionamentos.

\section{CONSIDERAÇÕES FINAIS}

Esta investigação apresenta a relação existente entre as experiências de violência na família de origem e o envolvimento em uma conjugalidade violenta na vida adulta. Ainda que a força da correlação não seja expressiva, as variáveis mostram-se relacionadas, em alguma medida, apontando a relevância de considerar as experiências do sujeito em sua família de origem, além de outros aspectos envolvidos no fenômeno da violência no conjugal. A família, como o primeiro laboratório de relações interpessoais em que o ser humano é envolvido, parece oferecer as ferramentas que o auxiliarão no estabelecimento de relações na vida adulta. Diante disso, vivenciar, como vítima ou como 
testemunha, violência familiar na infância oferece ao sujeito um modelo a ser perpetuado, ainda que seja gerador de dor e um legado de sofrimento. Nesse sentido, promover, de modo preventivo e terapêutico, a interrupção de ciclos conjugais de violência apresentase como fator protetor não somente para o núcleo familiar atual, mas também para as gerações futuras.

Esta pesquisa não tem a pretensão de generalizar os casos existentes, sendo uma limitação a ser considerada. Soma-se a isso, o fato de ter tido o foco na realidade local, sugerindo-se, assim, sua replicação em outros contextos sociais, a fim de explorar o fenômeno em estudo e ampliar dados para a área do conhecimento a que se encontra vinculado. Ainda, em se tratando de um tema tabu, cabe considerar a possibilidade da limitação a partir do autorrelato dos participantes. É possível ainda, que os dados sejam subnotificados, considerando os aspectos legais envolvidos nos contextos de violência conjugal, bem como os aspectos de desejabilidade social.
O presente estudo tem a intenção de fomentar questionamentos e incentivar a realização de futuras investigações acerca do tema, com metodologias e delineamentos diversos. Contudo, busca-se destacar a relevância do fenômeno da violência em sua característica transgeracional, bem como a importância de considerar o sofrimento causado a todos os envolvidos nas relações violentas; não apenas ao casal, mas também aos filhos, que experienciam esta realidade e podem levar para suas futuras relações afetivas o modelo relacional aprendido.

Com isso, destaca-se a relevância de políticas de atendimento a casais e famílias em situação de violência, bem como, o papel preventivo na educação baseados em resultados de pesquisas nacionais, com vistas a interromper a perpetuação de um padrão familiar violento. Assim, os serviços envolvidos nessas problemáticas podem contribuir com a promoção de saúde mental e qualidade de vida dos sujeitos envolvidos nos referidos contextos.

\section{REFERÊNCIAS}

Alexander, P. (2011). Childhood maltreatment, intimate partner violence, work interference and women's employment. Journal of Family Violence, 26(1), 255-261. https://dx.doi.org/10.1007/s10896-011-9361-9

Ansara, D. L. \& Hindin, M. J. (2009). Perpetration of intimate partner aggression by men and women in the Philippines: prevalence and associated factors. Journal of Interpersonal Violence, 24(9), 1579-1590. http://dx.doi. org/10.1177/0886260508323660

Banyard, V. L. \& Modecki, K. L. (2006). Interpersonal violence in adolescence: ecological correlates of self-reported perpetration. Journal of Interpersonal Violence, 21(10), 1314-1332. https://dx.doi.org/10.1177/0886260506291657

Black, D., Sussman, S., \& Unger, J. (2010). A further look at the intergenerational transmission of violence: witnessing interparental violence in emerging adulthood. Journal of Interpersonal Violence, 25(6), 1022-1042. https://dx.doi. org/10.1177/0886260509340539

Borsoi, T. S., Brandão, E. R., \& Cavalcanti, M. L. T. (2009). Ações para o enfrentamento da violência contra a mulher em duas unidades de atenção primária à saúde no município do Rio de Janeiro. Interface - Comunicação, Saúde, Educação, 13(28), 165-174. https://dx.doi.org/10.1590/S1414-32832009000100014

Boyle, D. J., O’Leary, K. D., Rosenbaum, A., \& Hassett-Walker, C. (2008). Differentiating between generally and partner-only violent subgroups: lifetime antisocial behavior, family of origin violence, and impulsivity. Journal of Family Violence, 23(1), 47-55. https://dx.doi.org/10.1007/s10896-007-9133-8

Bruschi, A., Paula, C. S., \& Bordin, I. A. S. (2006). Prevalência e procura de ajuda na violência conjugal física ao longo da vida. Revista de Saúde Pública, 40(2), 256-264. https://dx.doi.org/10.1590/S0034-89102006000200011

Carvalho-Barreto, A., Bucher-Maluschke, J. S. N. F., Almeida, P. C., \& DeSouza, E. (2009). Desenvolvimento humano e violência de gênero: uma integração bioecológica. Psicologia: Reflexão e Crítica, 22(1), 86-91. http://dx.doi. org/10.1590/s0102-79722009000100012

Cortez, M. B., Cruz, G. V., \& Souza, L. (2013). Violência conjugal: desafios e propostas para a aplicação da Lei Maria da Penha. Psico, 44(4), 499-507.

Dahlberg, L. D. \& Krug, E. G. (2007). Violência: um problema global de saúde pública. Ciência \& Saúde Coletiva, 11(Supl.), 1163-1178. https://dx.doi.org/10.1590/S1413-81232006000500007

Dourado, S. M. \& Noronha, C.V. (2014). A face marcada: as múltiplas implicações da vitimização feminina nas relações amorosas. Physis Revista de Saúde Coletiva, 24(2), 623-643. https://dx.doi.org/10.1590/S0103-73312014000200016

Durand, J. G., Schraiber, L. B., França-Junior, I., \& Barros, C. (2011). Repercussão da exposição à violência por parceiro íntimo no comportamento dos filhos. Rev. Saúde Pública, 45(2), 355-364. http://dx.doi.org/10.1590/S003489102011005000004

Falcke, D. (2003). Águas passadas não movem moinhos? As experiências da família de origem como preditoras da qualidade do relacionamento conjugal (Tese de Doutorado). Pontifícia Universidade Católica do Rio Grande do Sul, Porto Alegre, Brasil. 
Falcke, D.(2006). Filho de peixe, peixinho é: a importância das experiências na família de origem. Colóquio, 3(1), 83-97.

Fang, X. \& Corso, P. S. (2008). Gender differences in the connections between violence experienced as a child and perpetration of intimate partner violence in young adulthood. Journal of Family Violence, 23(5), 303-313. https:// dx.doi.org/10.1007/s10896-008-9152-0

Fehringer, J. A. \& Hindin, M. J. (2009). Like parent, like child: intergenerational transmission of partner violence in Cebu, the Philippines. Journal of Adolescent Health, 44(4), 363-371. https://dx.doi.org/10.1016/j.jadohealth.2008. 08.012 .

Fergusson, C. J. (2011). Love is a battlefield: risk factors and gender disparities for domestic violence among Mexican Americans. Journal of Aggression, Maltreatment \& Trauma, 20(2), 227-236. http://dx.doi.org/10.1016/j. chiabu.2005.10.006

Fernández-Montalvo, J., López-Go-i, J., \& Arteaga, A. (2011). Tratamiento de agresores contra la pareja en programas de atención a drogodependientes: un reto de future. Adicciones, 23(1), 5-9.

Gadoni-Costa, L. M., Zucatti, A. P. N., \& Dell'Aglio, D. D. (2011). Violência contra a mulher: levantamento dos casos atendidos no setor de psicologia de uma delegacia para a mulher. Estudos de Psicologia, 28(2), 219-227. http://dx.doi. org/10.1590/S0103-166X2011000200009

Garcia, M. V., Ribeiro, L. A., Jorge, M. T., Pereira, G. R., \& Resende, A. P. (2008) Caracterização dos casos de violência contra a mulher atendidos em três serviços na cidade de Uberlândia, Minas Gerais, Brasil. Cadernos de Saúde Pública, 24(11), 2551-2563. https://dx.doi.org/10.1590/S0102-311X2008001100010

Gover, A. R., Park, M., Tomsich, E. A., \& Jennings, W. G. (2011). Dating violence perpetration and victimization among south korean college students: a focus on gender and childhood maltreatment. Journal of Interpersonal Violence, 26(6), 1232-1263. https://dx.doi.org/10.1177/0886260508314330

Hughes, F. M., Stuart, G. L., Gordon, K. C., \& Moore, T. M. (2007). Predicting the use of aggressive conflict tactics in a sample of women arrested for domestic violence. Journal of Social and Personal Relationships, 24(2), 155-176. https://dx.doi.org/10.1177/0265407507075406

Jaoko, J. (2010). Correlates of wife abuse in the Maseno and Nairobi areas of Kenya. International Social Work, 53(1), 9-18. http://dx.doi.org/10.1177/0020872809348864

Kiss, L. B. \& Schraiber, L. B. (2011). Temas médico-sociais e a intervenção em saúde: a violência contra mulheres no discurso dos profissionais. Ciência \& Saúde Coletiva, 16(3), 1943-1952. http://dx.doi.org/10.1590/S141381232011000300028

Koenig, M. A., Stephenson, R., Ahmed, S., Jejeebhoy, S. J., \& Campbell, J. (2006). Individual and contextual determinants of domestic violence in North India. American Journal of Public Health, 96(1), 132-138. https://dx.doi.org/10.2105/ AJPH.2004.050872

Lamoglia, C. V. A. \& Minayo, M. C. S. (2009). Violência conjugal, um problema social e de saúde pública: Estudo em uma delegacia do interior do Rio de Janeiro. Ciência \& Saúde Coletiva, 14(1), 595-604. http://dx.doi.org/10.1590/ S1413-81232009000200028

Lawson, D. M. \& Rivera, S. (2008). Male partner abusers' perceptions of family relationship functioning: a comparison of clinically derived abuser types. Journal of Aggression, Maltreatment \& Trauma, 17(1), 59-79. https://dx.doi. org/10.1037/1524-9220.9.2.90

Leal, S. M. C., Lopes, M. J. M., \& Gaspar, M.F.M. (2011). Representações sociais da violência contra a mulher na perspectiva da enfermagem. Interface - Comunicação, Saúde, Educação, 15(37), 409-424. http://dx.doi.org/10.1590/ S1414-32832011005000012

Leôncio, K. L., Baldo, P. L., João, V. M., \& Biffi, R. G. (2008). O perfil de mulheres vitimizadas e seus agressores. Revista de Enfermagem UERJ, 16(3), 307-312.

Macy, R. \& Goodbourn, M. (2012). Promoting successful collaborations between domestic violence and substance abuse treatment service sectors: a review of the literature. Trauma, Violence \& Abuse, 13(4), 234-25. https://dx.doi. org/10.1177/1524838012455874

Martins, C., Ferriani, M. G., Silva, M. A., Zahr, N. R., Arone, K.M., \& Roque, E. M. S. (2007). A dinâmica familiar na visão de pais e filhos envolvidos na violência doméstica contra crianças e adolescentes. Revista Latino Americana de Enfermagem, 15(5).

Melchert, T. P. (1998a). A review of instruments for assessing family history. Clinical Psychology Review, 18, 163-187. http://dx.doi.org/10.1016/S0272-7358(97)00058-5

Melchert, T. P. (1998b). Testing the validity of an instrument for assessing family of origin history. Journal of Clinical Psychology, 54, 863-876.http://dx.doi.org/10.1002/(SICI)1097-4679(199811)54:7<863::AID-JCLP1>3.0.CO;2-G

Melo, Z. M., Silva, D. M., \& Caldas, M. T. (2009). Violência intrafamiliar: crimes contra a mulher na área metropolitana do Recife. Psicologia em Estudo, 14(1), 111-119. https://dx.doi.org/10.1590/S1413-73722009000100014

Mendlowicz, M. \& Figueira, I. (2007). Transmissão intergeracional da violência familiar: o papel do estresse póstraumático. Revista Brasileira de Psiquiatria, 29(1), 88-89. https://dx.doi.org/10.1590/S151644462007000100023

Milner, J. S., Thomsen, C. J., Crouch, J. L., Rabenhorst, M. M., Martens, P. M., Dyslin, C. W., Guimond, J. M., Stander, V. A., \& Merrill, L. L. (2010). Do trauma symptoms mediate the relationship between childhood physical abuse and adult child abuse risk? Child Abuse and Neglect, 34(5), 332-344. https://dx.doi.org/10.1016/j. chiabu.2009.09.017 
Miranda, M. P. M., Paula, C. S., \& Bordin, I. A. (2010). Violência conjugal física contra a mulher na vida: prevalência e impacto imediato na saúde, trabalho e família. Revista Panamericana de Salud Publica, 27(4), 300-308. http://dx.doi. org/10.1590/S1020-49892010000400009

Moraes, C. L., Hasselmann, M. H., \& Reichenheim, M. E. (2002). Adaptação transcultural para o português do instrumento "Revised Conflict Tactics Scales (CTS2)" utilizado para identificar a violência entre casais. Cadernos de Saúde Pública, 18(1), 163-175. http://dx.doi.org/10.1590/S0102-311X2002000100017

Mosmann, C., Zordan, E., \& Wagner, A. (2011). A qualidade conjugal como fator de proteção do ambiente familiar. In A. Wagner (Eds), Desafios psicossociais de família contemporânea (pp. 58-71). Porto Alegre: Artmed.

Narvaz, M. G. \& Koller, S. H. (2006). Mulheres vítimas de violência doméstica: compreendendo subjetividades assujeitadas. Psico, 37(1), 7-13.

Noll, J. G., Trickett, P. K., Harris, W. W., \& Putnam, F. W. (2009). The cumulative burden borne by offspring whose mothers were sexually abused as children: descriptive results from a multigenerational study. Journal of Interpersonal Violence, 24(3), 424-449. https://dx.doi.org/10.1177/0886260508317194

Padovani, R. \& Williams, L. (2011). Estilo parental de origem e ansiedade em homens com histórico de agressão à parceira. Estudos de Psicologia, 16(3), 263-269. https://dx.doi.org/10.1590/S1413-294X2011000300008

Paixão, G. P. N., Gomes, N. P., Diniz, N. M. F., Couto, T. M., Vianna, L. A. C., \& Santos, S. M. P. (2014). Situations which precipitate conflicts in the conjugal relationship: the women's discourse. Text Context Nursing, 23(4), 10411049. https://dx.doi.org/10.1590/0104-07072014003290013

Paradis, A. D. Reinherz, H. Z., Giaconia, R. M., Beardslee, W. R., Ward, K., \& Fitzmaurice, G. M.(2009). Long-term impact of family arguments and physical violence on adult functioning at age 30 years: findings from the simmons longitudinal study. Journal of American Academic Child and Adolescence Psychiatry, 48(3), 290-298. https://dx.doi. org/10.1097/CHI.0b013e3181948fdd

Pedro, M. M. F. (2013). Relação conjugal e relação pais-filhos: estudo de variáveis mediadoras e moderadoras (Tese de Doutorado). Universidade de Lisboa, Faculdade de Psicologia.

Pinto, A. S., Moraes, O. C. R., \& Monteiro, J.(Orgs.). (2015). Dossiê mulher 2015. Rio de Janeiro: Instituto de Segurança Pública.

Rosa, A., Boing, A., Büchele, F., Oliveira, W., \& Coelho E. (2008). A violência conjugal contra a mulher a partir da ótica do homem autor da violência. Saúde e Sociedade, 17(3), 152-160. http://dx.doi.org/10.1590/S0104-12902008000300015

Silva, I. M., Menezes, C. C., \& Lopes, R. C. S. (2010). Em busca da "cara-metade": motivações para a escolha do cônjuge. Estudos de Psicologia, 27(3), 383-391. https://dx.doi.org/10.1590/S0103-166X2010000300010

Silva, J. G., Valadares, F. C., \& Souza, E. R. (2013). O desafio de compreender a consequência fatal da violência em dois municípios brasileiros. Interface - Comunicação, Saúde, Educação, 17(46), 535-548. http://dx.doi.org/10.1590/ S1414-32832013005000022

Souto, C. M. R. M. \& Braga, V. A. B. (2009) Vivências da vida conjugal: posicionamento das mulheres. Rev. Bras. Enferm., 62(5), 670-674. http://dx.doi.org/10.1590/s0034-71672009000500003

Stenson, K. \& Heimer, G. (2008). Prevalence of experiences of partner violence among female health staff: relevance to awareness and action when meeting abused women patients. Women's Health Issues, 18(2), 141-149. http://dx.doi org/10.1016/j.whi.2007.12.003

Stickley, A., Timofeeva, I., \& Sparen, P. (2008). Risk factors for intimate partner Violence against women in St. Petersburg, Russia. Violence Against Woman, 14(4), 483-495. https://dx.doi.org/10.1177/1077801208314847

Straus, M. (2010). Thirty years of denying the evidence on gender symmetry in partner violence: implications for prevention and treatment. Partner Abuse, 1(3), 332-363. https://dx.doi.org/10.1891/1946-6560.1.3.332

Straus, M., Hamby, S., McCoy, S. B., \& Sugarman, D. (1996). The Revised Conflict Tactics Scales (CTS2): Development and preliminary psychometric data. Journal of Family Issues, 17(3), 283-316. http://dx.doi.org/ $10.1177 / 019251396017003001$

Vieira, E. M., Perdona, G. S. C., \& Santos, M. A. (2011). Fatores associados à violência física por parceiro íntimo em usuárias de serviços de saúde. Revista de Saúde Pública, 45(4), 730-737. https://dx.doi.org/10.1590/S003489102011005000034

Villas Boas, A. C. V. B., Dessen, M. A., \& Melchiori, L. E. (2010). Conflitos conjugais e seus efeitos sobre o comportamento de crianças: uma revisão teórica. Arquivos Brasileiros de Psicologia, 62(2), 91-102.

Wagner, A., Goulart, V. (2013). Os conflitos conjugais na perspectiva dos filhos. Arquivos Brasileiros de Psicologia, 65(3), 392-408.

Weisbart, C. E., Thompson, R., Pelaez-Merrick, M., Kim, J., Wike, T., Briggs, E., English, D. J., \& Dubowitz, H. (2008). Child and adult victimization: sequelae for female caregivers of high-risk children. Child Maltreatment, 13(3), 235-244. https://dx.doi.org/10.1177/1077559508318392

Whiting, J. B., Simmons, L. A., Havens, J. R., Smith, D. B., \& Oka, M. (2009). Intergenerational transmission of violence: the influence of self-appraisals, mental disorders and substance abuse. Journal of Family Violence, 24(8), 639-648. https://dx.doi.org/10.1007/s10896-009-9262-3

Yoshihma, M. \& Horrocks, J. (2010). Risk of intimate partner violence: role of childhood sexual abuse and sexual initiation in women in Japan. Children and Youth Services Review, 32(1), 28-37. http://dx.doi.org/10.1016/j. childyouth.2009.06.013 
Zanoti-Jeronymo, D. V., Zalesky, M., Pinsky, I., Caetano, R., Fliglie, N. B., \& Laranjeira, R. (2009). Prevalência de abuso físico na infância e exposição à violência parental em uma amostra brasileira. Cadernos de Saúde Pública, 25(11), 2467-2479. https://dx.doi.org/10.1590/S0102-311X2009001100016

Autores:

PATRÍCIA MANOZZO COLOSSI - Doutoranda em Psicologia Clínica (UNISINOS).

Aline Riboli MarasCA - Psicóloga, Universidade do Vale do Rio dos Sinos (UNISINOS).

DENISE FALCKE - Doutora em Psicologia, Universidade do Vale do Rio dos Sinos (UNISINOS).

Endereço para correspondência:

Patrícia Manozzo Colossi

Av. General Barreto Viana, 1175/1106

CEP 91330-630 Porto Alegre, RS, Brasil

E-mail: pmcolossi@gmail.com

Aceito em: 04.06.15

Recebido em: 14.10 .15 